ARTICLE

\title{
Density Matrix Renormalization Group and Numerical Diagonalization Study on the Quantum Spin Nanotube in Magnetic Field
}

\author{
Toru SAKAI ${ }^{1, *}$, Masahiro SATO ${ }^{2}$, Kouichi OKUNISHI ${ }^{3}$, \\ Kiyomi OKAMOTO $^{4}$ and Chigak ITOI $^{5}$ \\ ${ }^{1}$ Japan Atomic Energy Agency, SPring-8, Hyogo 679-5148, Japan \\ and Department of Material Science, University of Hyogo, Hyogo 678-1297, Japan \\ and JST TRIP, Saitama 332-0012, Japan \\ ${ }^{2}$ Aoyama Gakuin University, Sagamihara,Kanagawa 251-5258, Japan \\ ${ }^{3}$ Depatment of Physics, Niigata University, Niigata, Japan \\ ${ }^{4}$ Department of Physics, Tokyo Institute of Technology, Tokyo 152-8551, Japan \\ ${ }^{5}$ Department of Physics, Nihon University, Tokyo 101-8308, Japan
}

\begin{abstract}
The magnetization process of the $S=1 / 2$ three-leg spin tube with an asymmetric exchange interaction in each triangle unit is investigated by the numerical exact diagonalization and the density matrix renormalization group calculation. It is found that the 1/3 magnetization plateau appears due to two different mechanisms depending on the asymmetry. The phase diagrams of the $1 / 3$ plateau is presented. In addition some cusp-like anomalies are found in the magnetization curve.
\end{abstract}

KEYWORDS: spin nanotube, density matrix renormalization gourp, numerical diagonalization, spin gap, spin liquid, chirality

\section{Introduction}

The spin nanotube is one of interesting nanomaterials which are expected to be developed towards some functional devices in the near future, like the carbon nanotube. Since the $\mathrm{S}=1 / 2$ three-leg spin tube $\left[\left(\mathrm{CuCl}_{2} \text { tach } \cdot \mathrm{H}\right)_{3} \mathrm{Cl}\right] \mathrm{Cl}_{2}$ was synthesized, ${ }^{1)}$ several theoretical works about this systems have been published. ${ }^{2-11)}$ In our previous theoretical work ${ }^{7)}$ the spin gap was revealed to be very fragile against the lattice distortion from the regular triangle to the isosceles one, and the Berezinskii-Kosterlitz-Thouless (BKT) quantum phase transition $^{12,13)}$ was predicted from the gapped to the gapless phases with respect to such an asymmetry of the rung exchange interactions.

On the other hand, the numerical exact diagonalization study indicated the existence of the $1 / 3$ magnetization plateau for sufficiently large regular triangle exchange interaction. ${ }^{8)}$ Thus it would be also interesting to investigate the magnetization process of the isosceles triangle spin tube, particularly how the asymmetry affects the $1 / 3$ plateau. In this paper, we consider two different mechanisms of the $1 / 3$ plateau and present the phase diagram using the numerical exact diagonalization analysis. A new phase is revealed to appear between the two different mechanisms. In addition some cusp-like anomalies are found by the density matrix renormalization group (DMRG) approach. ${ }^{14,15)}$

\section{Model}

We consider the $S=1 / 2$ asymmetric three-leg spin tube,

*Corresponding author, E-mail: sakai@spring8.or.jp

(C) 2011 Atomic Energy Society of Japan, All Rights Reserved.

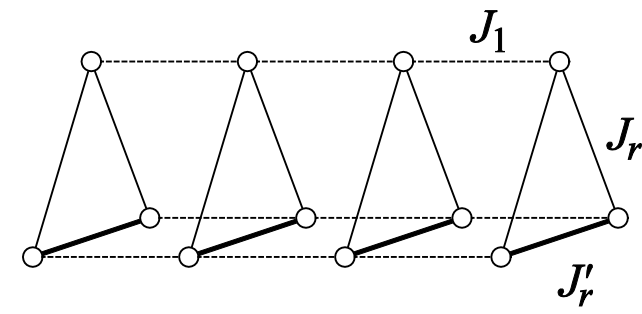

Fig. 1 Structure of the three-leg asymmetric spin tube (1)

shown in Fig. 1, described by the Hamiltonian

$$
\begin{aligned}
\hat{H}= & J_{1} \sum_{i=1}^{3} \sum_{j=1}^{L} \vec{S}_{i, j} \cdot \vec{S}_{i, j+1}+J_{r} \sum_{i=1}^{2} \sum_{j=1}^{L} \vec{S}_{i, j} \cdot \vec{S}_{i+1, j} \\
& +J^{\prime}{ }_{r} \sum_{j=1}^{L} \vec{S}_{3, j} \cdot \vec{S}_{1, j},
\end{aligned}
$$

where $\vec{S}_{i, j}$ is the spin- $1 / 2$ operator and $L$ is the length of the tube in the leg direction. The exchange interaction constant $J_{1}$ is for the neighboring spin pairs along the legs, while $J_{\mathrm{r}}$ and $J_{\mathrm{r}}^{\prime}$ are the rung interaction constants. All the exchange interactions are supposed to be antiferromagnetic (namely, positive).

The ratio $\alpha=J_{\mathrm{r}}^{\prime} / J_{\mathrm{r}}$ stands for the degree of the asymmetry of the rung interactions. We will vary $\alpha$ and $J_{1}$ to investigate the quantum phase transitions. Throughout this paper, we fix $J_{\mathrm{r}}$ to one. The effect of the magnetic fields is incorporated by adding the Zeeman energy 


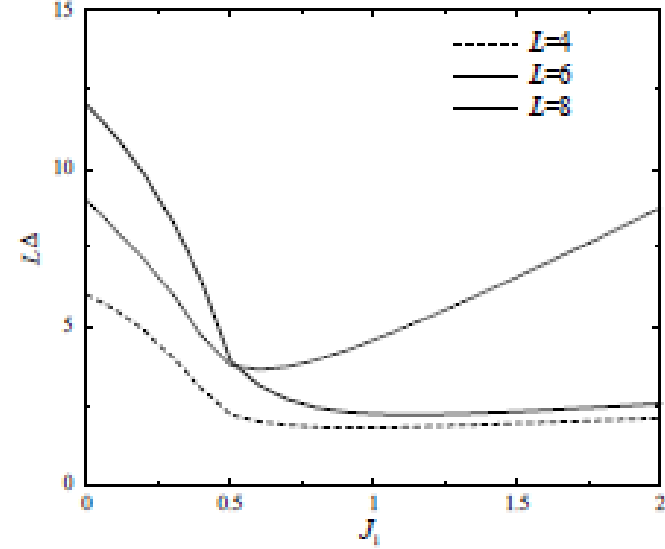

Fig. 2 Scaled gaps for $L=4,6$ and 8 plotted versus $J_{1}$ with fixed $J_{\mathrm{r}}^{\prime} / J_{\mathrm{r}}=1$

$$
\hat{H}_{Z}=-H \sum_{i=1}^{3} \sum_{j=1}^{L} S_{i, j}^{z} .
$$

The present model includes three typical models as limiting cases; (a) $\alpha=0$ : the three-leg spin ladder, (b) $\alpha=1$ : the symmetric spin tube, and (c) $\alpha \rightarrow \infty$ : the single chain plus rung dimers. Since the system is gapless in the cases (a) and (c), while gapful in the case (b), at least two quantum phase transitions should occur with increasing $\alpha$ from 0 to infinity. As we showed in the previous works, ${ }^{6,10)}$ the one-site translational symmetry along the $\operatorname{leg}\left(\vec{S}_{i, j} \rightarrow \vec{S}_{i, j+1}\right)$ is spontaneously broken in the symmetric spin tube at least in the strong-rung-coupling regime.

\section{1/3 Magnetization Plateau}

In order to study the $1 / 3$ magnetization plateau, we calculated the spin excitation gap at $m / m_{s}=1 / 3 \quad(m$ is the magnetization and $m_{\mathrm{s}}$ is the saturation magnetization), namely

$$
\Delta=E(M+1)+E(M-1)-2 E(M),
$$

where $E(M)$ is the lowest energy in the subspace $\sum S_{i, j}^{z}=M$ and $M=L / 2$, using the numerical exact diagonaliziation. The scaled gap $L \Delta$ for $\alpha=1$ is plotted versus $J_{1}$ for $L=4,6$ and 8 in Fig. 2. From the cross point between $L=6$ and 8 , the quantum critical point is estimated as $J_{1} \sim 0.5$ between the plateau and gapless (no plateau) phases.

In comparison with the spin gap at $H=0$, the $1 / 3$ plateau is expected to be stable against the asymmetry $(\alpha \neq 1)$, because it was also predicted to exist even for the three-leg ladder $(\alpha$ $=0)$.

At the $1 / 3$ plateau phase close to $\alpha=0$, the state $\mid \uparrow \downarrow \uparrow$, is expected to be stabilized at each triangle unit, while |singlet, $\uparrow$, for the other limit $\alpha \rightarrow \infty$. We call the two mechanisms of the plateau formation as the udu mechanism and dimer-monomer mechanism, respectively. Thus the three phases; the udu plateau, the dimer-monomer plateau and gapless phases are expected to occur at $m / m_{\mathrm{s}}=1 / 3$.

Next we consider the boundary between the two different plateau phases. It is expected to appear around $\alpha=1$ and $J_{1}<0.5$. The $\alpha$ dependences of the plateau width estimated

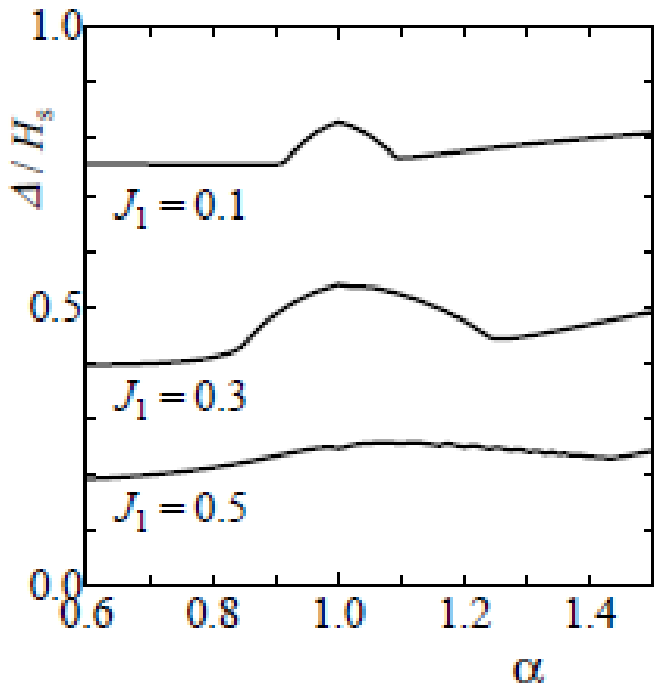

Fig. $3 \alpha$ dependence of the plateau width normalized by the saturation magnetic field $H_{\mathrm{s}}$ calculated by DMRG for $J_{1}=0.1,0.3$ and 0.5

by the DMRG calculation for $L=128$ for $J_{1}=0.1,0.3$, and 0.5 are shown in Fig. 3. Figure 3 indicates clear anomalous increases of the plateau width for $J_{1}=0.1$ and 0.3 . The anomalous increase region is supposed to be a new phase between the two plateau phases. In order to examine the properties of the new phase, the lowest excitation gap within the $\mathrm{M}=\mathrm{M}_{\mathrm{s}} / 3$ space, $\Delta_{0}$, with $k=\pi$ and the odd parity for the inversion with respect to the center line of the isosceles triangle, is calculated by the numerical diagonalization. We note that the excitation gap (or magnetization plateau width) $\Delta$ from the $M=M_{s} / 3$ state to $M=M_{s} / 3 \pm 1$ states is always finite when $\alpha$ is varied. The scaled excitation gaps $L \Delta_{0}$ for $L=4,6$, and 8 are plotted versus $\alpha$ with fixed $J_{1}=0.4$ in Fig. 4 . It indicates that the excited state is degenerated with the ground state around $0.9 \sim \alpha \sim 1.1$, which is expected to correspond to the anomalous increase region in Fig. 3. It suggests that the doubly degenerate state is realized in this region. In fact our preliminary DMRG calculation shows the existence of the spontaneous breaking of the translational and inversion symmetries and the long-range chiral order.

\section{Phase Diagram}

The phase boundary of the new plateau phase can be determined by the phenomenological renormalization applied for the scaled gap $L \Delta_{0}$, using the relation,

$$
(L+2) \Delta_{0, L+2}\left(\alpha_{c, L}\right)=L \Delta_{0, L}\left(\alpha_{c, L}\right),
$$

where $\alpha_{c, L}$ is the size-dependent fixed point. The fixed points $\alpha_{c, L}$ for $L=6$ (dashed lines) are shown in the $\alpha-J_{1}$ plane of Fig. 5. The solid line is the plateau-plateauless phase boundary estimated by the phenomenological renormalization for the scaled gap $L \Delta$. In the phase diagram in Fig. 5, the plateau 1, 2 and 3 correspond to the udu, the dimer-monomer, and the new plateau phases, respectively. In the present phase diagram the dashed lines are the second-order boundaries, while the solid curves are expected to be of the BKT tran- 


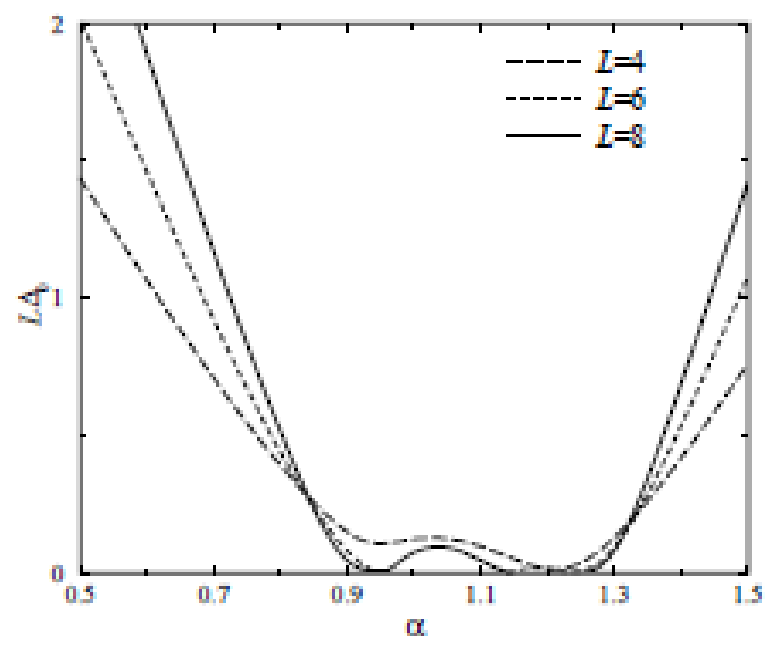

Fig. 4 Scaled gaps $L \Delta_{0}$ for $L=4,6$ and 8 plotted versus $\alpha$ with fixed $J_{1}=0.4$

sition. According to the comparison with the result of $L=4$, the second-order phase boundary is expected to have a few percent of the finite-size correction at most, while the BKT line still has a much larger error. In a previous paper, ${ }^{7)}$ we have succeeded to obtain the phase diagram in the absence of magnetic field by use of the effective theory starting from the Hubbard model. We have also applied this method to the $\mathrm{M}_{\mathrm{s}} / 3$ plateau problem, by which the phase diagram of Fig. 5 was qualitatively reproduced.

\section{Magnetization Curves}

Finally we present the magnetization curves calculated by the DMRG for $\alpha=0.5,1.0$ and 1.5 with fixed $J_{1}=1$ in Fig. 6 . Since the $1 / 3$ plateau appears for $\alpha=1.0$, the true tricritical point should be at larger $J_{1}$ than that in Fig. 5 . Some precise finite-size scaling analyses would be necessary.

The magnetization curve for $\alpha=1.5$ in Fig. 6 exhibits a cusp-like anomaly about $H \sim 3$, which is possibly another field-induced quantum phase transition. It would be interesting to identify it.

\section{Summary}

The magnetization process of the $S=1 / 2$ three-leg spin tube was investigated by the numerical exact diagonalization and DMRG method. We consider the two mechanisms of the $1 / 3$ plateau for the isosceles triangle tube and present the phase diagram. The DMRG analysis also indicated a cusp-like anomaly in the magnetization curve.

\section{Acknowledgement}

This work has beenpartly supported by Grants-in-Aid for Scientific Research (B) (No.17340100, No.20340096), Scientific Research (C) (No.18540340) and Priority Areas "Invention of Anomalous Quantum Materials --- New Physics through Innovation Materials ---" (No.19014019), "Physics of New Quantum Phases in Superclean Materials" (No.17071011, No.18043023, No.20029020) and "High Field Spin Science in 100T" (No.20030008, No.2003003) from the Ministry of Education, Culture, Sports, Science and

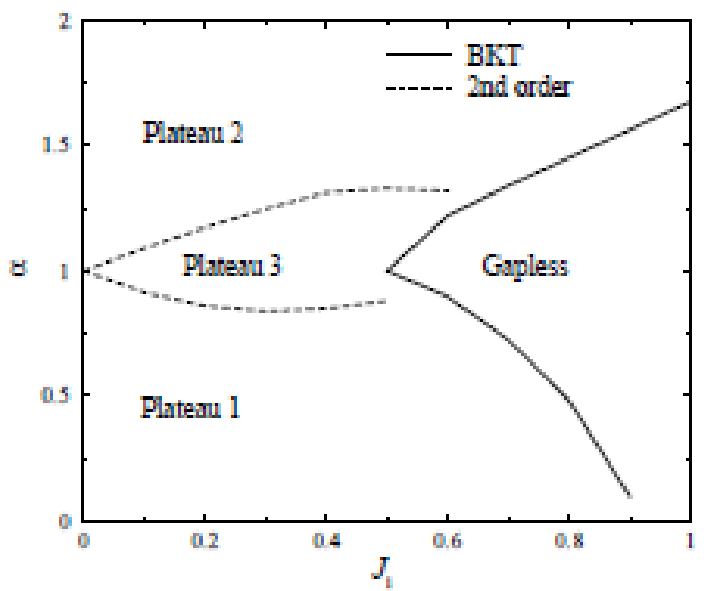

Fig. 5 S Phase diagram of the $1 / 3$ magnetization plateau

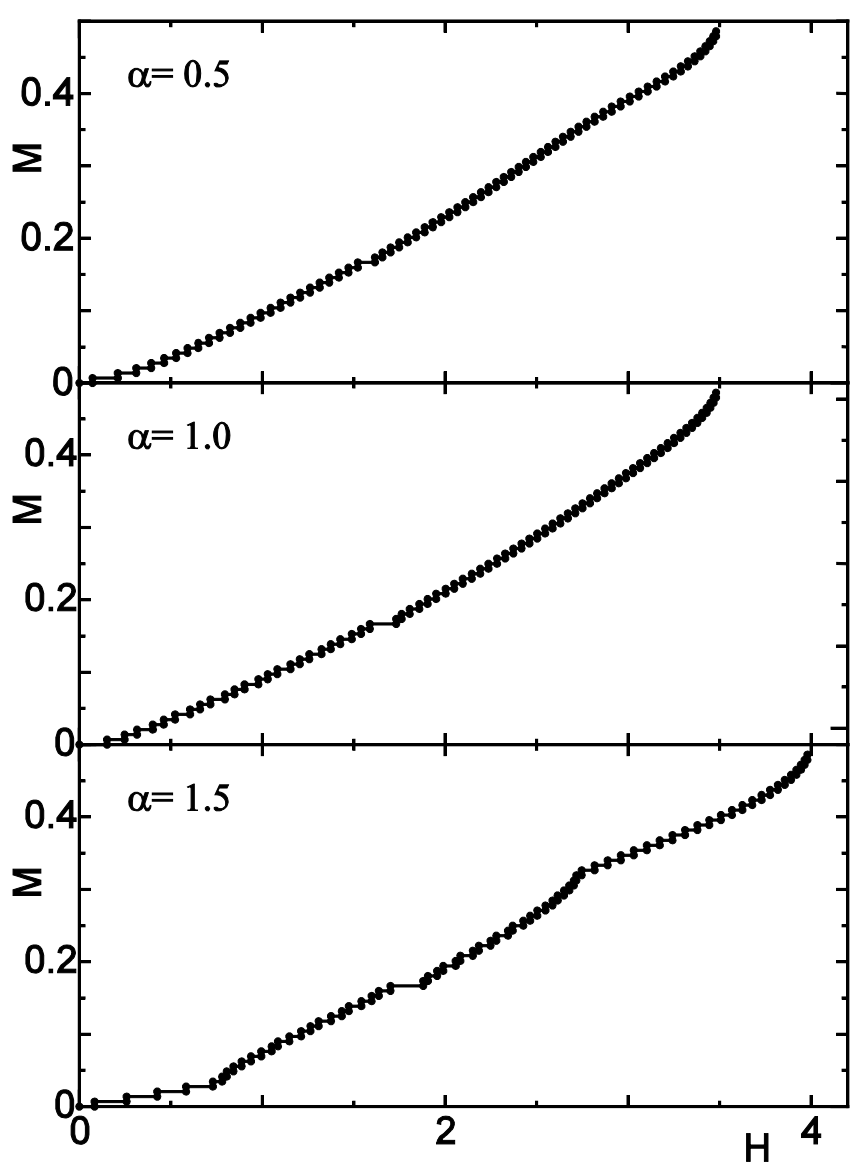

Fig. 6 Magnetization curves calculated by DMRG for $\alpha=0.5,1.0$ and 1.5

Technology of Japan. We further thank the Supercomputer Center, Institute for Solid State Physics, University of Tokyo, the Cyberscience Center, Tohoku University, and the Computer Room, Yukawa Institute for Theoretical Physics, Kyoto University for computational facilities.

\section{References}

1) J. Schnack, H. Nojiri, P. Koegerler, G. J. T. Cooper, L. Cronin, Phys. Rev., B70, 174420 (2004). 
2) T. Sakai, M. Matsumoto, K. Okunishi, K. Okamoto, M. Sato, Physica, E29 633 (2005).

3) J.-B. Fouet, A. Laeuchli, A. Pilgram, R. M. Noak, F. Mila, Phys. Rev., B73, 014409 (2006).

4) K. Okunishi, S. Yoshikawa, T. Sakai, S. Miyashita, Prog. Theor. Phys. Suppl., 159, 297 (2005).

5) M. Sato, T. Sakai, Phys. Rev., B75, 014411 (2007).

6) S. Nishimoto, M. Arikawa, Phys. Rev., B78, 054421 (2008).

7) T. Sakai, M. Sato, K. Okunishi, Y. Otsuka, K. Okamoto, C. Itoi, Phys. Rev., B78, 184415 (2008).

8) D. C. Cabra, A. Honecker, P. Pujol, Phys. Rev., B58, 6241 (1998).
9) H. J. Schulz, in Correlated Fermions and Transport in Mesoscopic Systems, (ed.) T. Martin, G. Montambaux, J. Tran Than Van (1996); arXive:9605075.

10) K. Kawano, M. Takahashi, J. Phys. Soc. Jpn., 66, 4001 (1997).

11) A. Luscher, R. M. Noack, G. Misguich, V. N. Kotov, F. Mila, Phys. Rev., B70, 060405(R) (2004).

12) V. L. Berezinskii, Zh. Eksp. Teor. Fiz., 61, 1144 (1971) (Sov. Phys. JETP, 34, 610 (1972)).

13) J. M. Kosterlitz, D. J. Thouless, J. Phys., C6, 1181 (1973).

14) S. R. White, Phys. Rev. Lett., 69, 2863 (1992).

15) S. R. White, Phys. Rev., B48, 10345 (1993). 